\title{
Mahrouguetien, Khanguet El-Mahrouguet (Préhistoire)
}

C. Roubet

\section{(2) OpenEdition}

1 Journals

Édition électronique

URL : http://journals.openedition.org/encyclopedieberbere/414

DOI : 10.4000/encyclopedieberbere.414

ISSN : 2262-7197

Éditeur

Peeters Publishers

\section{Édition imprimée}

Date de publication : 29 décembre 2010

Pagination : 4501-4508

ISBN : 978-90-429-2367-6

ISSN : 1015-7344

Référence électronique

C. Roubet, "Mahrouguetien, Khanguet El-Mahrouguet (Préhistoire) », Encyclopédie berbère [En ligne], 30 | 2010, document M15, mis en ligne le 17 septembre 2020, consulté le 12 octobre 2020. URL : http://journals.openedition.org/encyclopedieberbere/414; DOI : https://doi.org/10.4000/ encyclopedieberbere.414

Ce document a été généré automatiquement le 12 octobre 2020.

(c) Tous droits réservés 


\title{
Mahrouguetien, Khanguet El- Mahrouguet (Préhistoire)
}

\author{
C. Roubet
}

Depuis sa découverte dans les Némencha, le «Mahrouguétien", robuste mobilier macrolithique à retouche bifaciale, déconcerte les chercheurs, en Algérie. Mélanges et contaminations furent d'emblée invoqués. Une attribution au Paléolithique ou au Néolithique fut alternativement proposée. Voilà près de 70 ans qu'on hésite encore. De quoi s'agit-il? Il s'agit de grandes pièces épaisses, peu retouchées. Rien dans leur façonnage ne relève de répertoires techniques appliqués au Néolithique, d'ascendance épipaléolithique. Par d'autres de leurs traits, ces pièces sont aussi très éloignées de l'Acheuléen bifacial paléolithique. Aucune analyse à ce jour ne leur a été consacrée. En dépit de découvertes abondantes mais anciennes, sur plusieurs sites de plein air, privés d'autres marqueurs culturels, a-t-on dit, un injustifiable discrédit subsiste.

\section{Activités agricoles probables : houes et pics du Mahrouguétien}

2 A plus d'une centaine de kilomètres au sud de Tébessa, au delà d'El Ma-el-Abiod, se dresse le Djebel el Mahrouga à 1070m d'alt. et coule l'Oued Mahrouguet, affluent de rive droite de l'Oued Brisgane. C'est là que se situerait l'emplacement d'un assemblage macrolithique, bifacial, nommé "Mahrouguétien ». Cette découverte et cette création terminologique faites par M. Reygasse, remontent à 1934 (1936-1937). Aucune de ces brèves notes ne renferme d'informations précises, de renseignements topographiques, ni de récit de la découverte. La carte de Tébessa (IGN, feuille de Fériana, $\mathrm{n}^{\circ} 40$ au 1 : 200 000, révision 1934) signalait déjà ce massif-repère, qui resta pour les autochtones l'effrayant massif brûlé ou Dj. el Mahrouga; la toponymie berbère a conservé l'ancestrale mémoire d'une terreur, née d'un grandiose incendie, qui dut, un jour, ravager et calciner les forêts de ce massif. Ce lieu, non habité, devenu maigre terre de pâturage, subit aujourd'hui les effets d'une désertification, mais ne semble pas avoir connu d'importantes modifications liées au tracé des voies de communications reliant 
El Ma-el-Abiod à Bir Sbeïkia ; les environs sont porteurs d'une mémoire qui mériterait d'être recherchée, lors de nouvelles prospections.

31934 : Privés d'historique, rappelons brièvement les propos de M. Reygasse (1934): "Aux environs du Khanguet el Mahrouguet, on trouve en place, de nombreux bancs de silex dont les rognons ont été utilisés... M. Breuil estime que les formes pics, ciseaux, tranchets, s'expliqueraient par un déterminisme indusindustriel», ailleurs il ajoutera: "J'ai enfin relevé dans ce même lieu de beaux éclats très caractéristiques de la technique clactonienne. Ces éclats à l'Oued Mahrouguet accompagnent toujours les pièces de technique acheuléenne et campignienne, caractéristiques de ce milieu » (Reygasse 1936-1937). Il faut reconnaître que le soutien de l'Abbé apporté à $\mathrm{M}$. Reygasse, en dialoguant et en étudiant à ses côtés, au Musée du Bardo d'Alger, les collections qui venaient d'y être transportées, aurait pu nourrir les publications d'informations plus pertinentes.

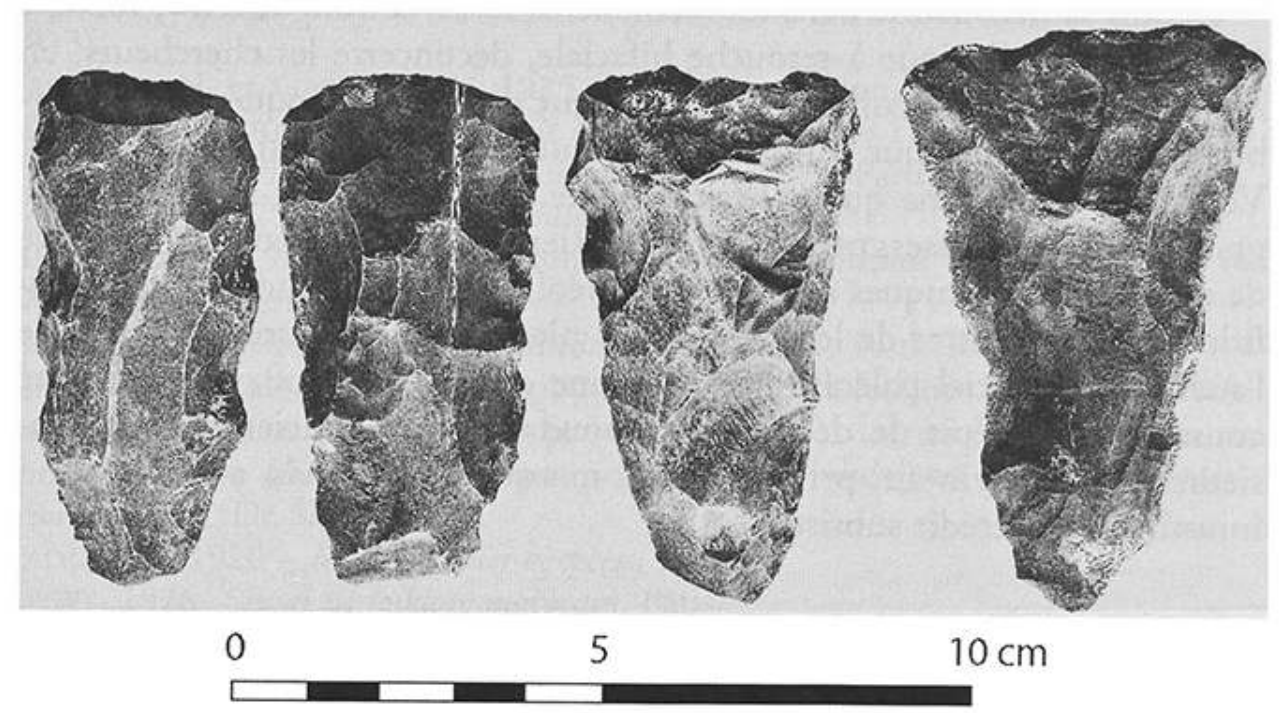

Fig. 1. Floues du mahrouguétien, PROVEnANT de L'OUEd MAHROUgUet, (SUd d'el-MA EL-ABIOD, ALgÉRIE). DÉCOUVERTE M. REYgASSE, 1934

Cl. M. Reygasse, 1934.

4 Ambiguïtés et confusions, issues d'une connaissance alors élémentaire de la technologie paléolithique, troublèrent le jugement des premiers experts d'un Acheuléen sur silex, disposant des documents comparatifs d'El Ma-el-Abiod, de facture exceptionnelle, antérieurement découverts dans cette région (Debruge 1909, Reygasse 1911).

51955 : On doit à L. Balout (1955), le diagnostic définitif réfutant le rattachement de ce matériel au Paléolithique inférieur et à l'Acheuléen du Constantinois. Depuis longtemps déjà on avait souligné, dit-il, l'apparition et le développement en France de tels instruments lourds dans les contextes néolithiques, qualifiés de Néolithique des Plateaux. Citons L. Balout, p. 452 : «On doit ajouter l'existence d'un faciès à gros outillage, d'allure faussement paléolithique, avec des formes qui, comme les pics évoquent le Campignien d'Europe, et qui est encore extrêmement mal connu. Quelle est la place chronologique du "Mahrouguétien » de Reygasse ?... J'ai entendu nommer « Néolithique des Plateaux» en France, un autre ensemble dont certaines pièces ont un faux air paléolithique ». «Il n'est pas impossible que nous ayons là un faciès du Néolithique tardif ».

Plus aucune autre remarque ne fut portée sur ces documents. 
72005 : Tenter de donner aujourd'hui une nouvelle substance à la Néolithisation: rechercher les traces de probables travaux agricoles dans les Némencha, puis, en se penchant sur une vielle carte, comprendre en tenant en main de primitives « houes en pierre ", jadis façonnées au Khanguet el Mahrouguet que subsiste l'unique témoignage d'un magistral évènement qui bouleversa les hommes d'alors, mais lesquels? Quand ? Tel est le projet qui motive aujourd'hui l'archéologue.

Quand on regarde pour la première fois cette documentation, son caractère typique, livre au premier coup d'œil un diagnostic qui peut être aujourd'hui précisé. Cet assemblage en silex fut façonné sur place. Avant tout ramassage, il était resté homogène, typé et numériquement important. Comment le pressentir? En m'appuyant sur un examen général des objets récoltés, qui gisent aujourd'hui «dévitalisés » et accumulés en portons, dans les meubles des Réserves de nos institutions (IPH-MNHN, Paris), il me semble possible de retrouver, mentalement, certains aspects. Pourtant, au moment de leur découverte $M$. Reygasse aurait pu observer les caractéristiques particulières d'une répartition au sol. Par endroits au moins, cet assemblage dut apparaître, tantôt éparpillé, tantôt groupé. Non loin de zones protégées (rochers épars, végétation), les objets les plus lourds durent se maintenir en place, sur des aires peu modifiées par les intempéries; là, devaient s'être conservés sans doute, quelques témoins figés d'une ultime phase au moins d'un atelier actif. Peu avant les ramassages, chaque pièce au sol aurait pu avoir-en partie-conservé la dernière place d'un état de l'activité, avant l'abandon. Mais, les ramassages quantitatifs, sélectifs et successifs, rapidement faits sans carroyage, ni photo, puis le tri et la dissociation des pièces en catégories identifiables, suivie d'une ultime sélection au profit des institutions d'Alger, le CRAPE-CNRPAH et de Paris l'IPH-MNHN, firent perdre à tous les objets leur potentielle et initiale cohésion, leur forte liaison, leur appartenance à une aire précise. C'est ainsi que pourrait avoir disparu le dernier lien avec le sol, suggestif d'un ensemble témoin d'activités spécialisées et connectées. Tout archéologue sait que cette information fondamentale que livre le terrain ne se déduira pas des plus subtiles analyses. Pas même d'éventuels raccords que la patience pourrait permettre d'obtenir.

Quoi, qu'il en soit, il existe bien, pour moi, un « Ensemble Mahrouguétien » d'âge tardif, probablement Néolithique, mais pas encore un faciès comme suggéré par M. Reygasse. Nombre de documents typés l'attestent, comme ces macro-éclats bruts, témoins d'un débitage à la pierre in situ. Les pièces achevées, restées intactes, (Fig. 2) paraissent correspondre à des outils expédients, sommairement façonnés, qui n'auraient peut-être pas exigé un long investissement technique. Ce façonnage sobre et simple est parvenu à donner a l'ébauché le caractère net de l'outil en préparation, sa spécificité, qu'une finition par retouche écailleuse dut ensuite affiner et renforcer. Quant à envisager la présence de probables morphologies aratoires de houes et de pics, encore faudrait-il envisager que ces pièces aient été préalablement emmanchées pour que des comparaisons avec d'analogues objets de pierre africains, devenus familiers, nous préparent à retenir cette hypothèse (Vaufrey 1969). 


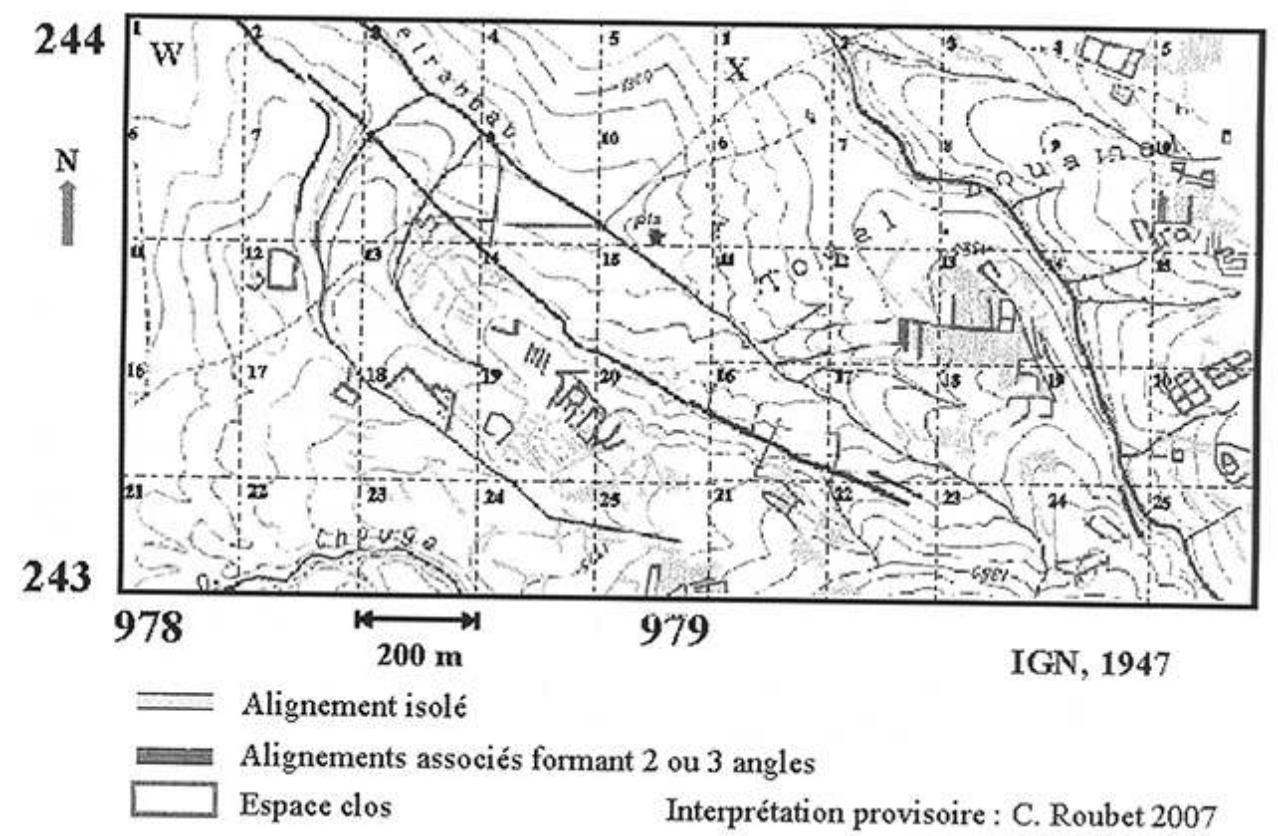

Fig. 2. "QuAdrillage de tAZBent ", ZONe 23-24 OU WX, ChAQUe CARRÉ MESURE 1 KM². PREMière ANALYSE DES ALIGNEMENTS, COMPARTIMENTS ET STRUCTURES RELEVÉS PAR LES INgÉNIEURS gÉOgRAPHES DE L'IGN, 1947.

Carrés $\mathrm{J}-\mathrm{K}: \mathbf{2} \mathrm{km}^{2}$

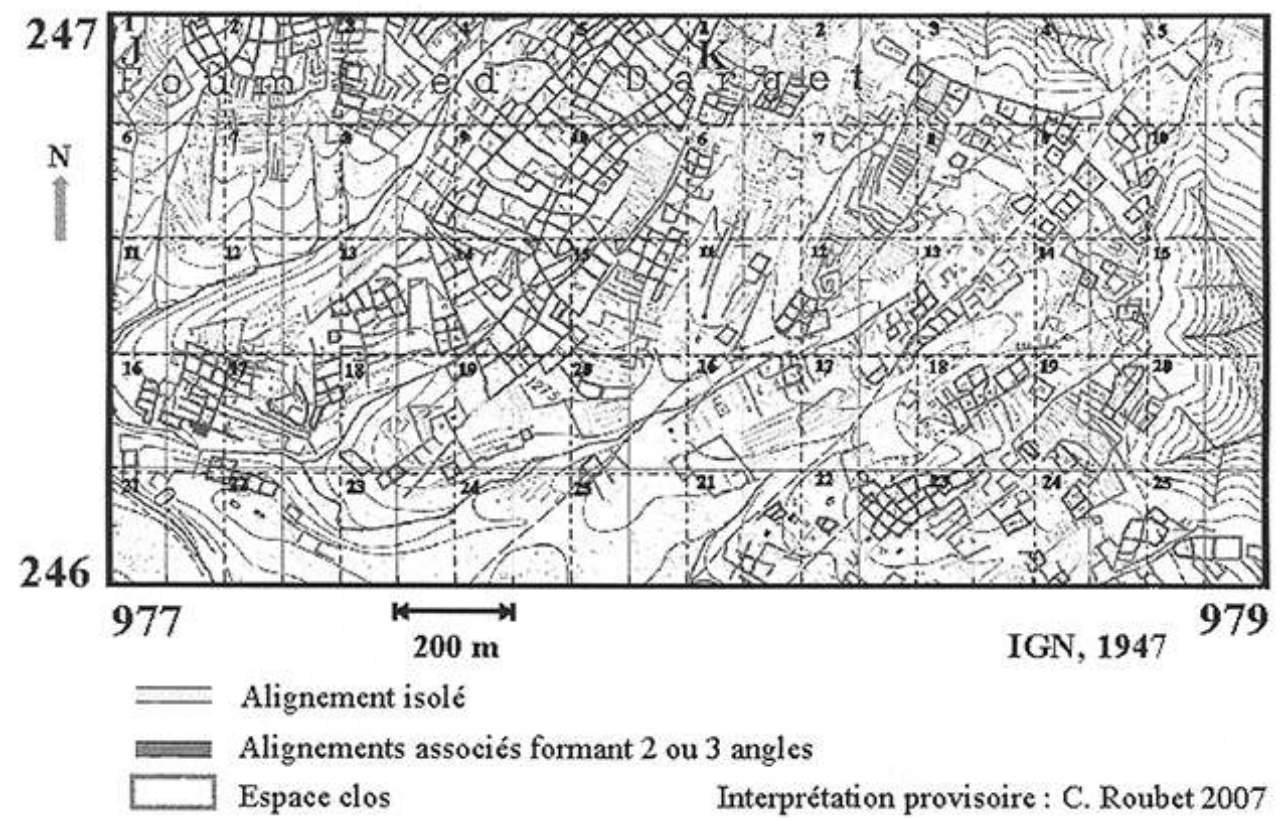

FIg. 3. " QUADRILLAge DE TAZBENT ", ZONE 10-11 OU JK, CHAQUE CARRÉ MESURE 1 KM². PREMIÈre ANALYSE DES ALIgNEMENTS, COMPARTIMENTS ET STRUCTURES RELEVÉS PAR LES INgÉNIEURS gÉogRAPHES DE L'IGN, 1947.

Des études appropriées définiront cet ensemble industriel qui pourrait bien avoir conservé de très primitifs instruments pré-agricoles. Mentionnons ici qu'aucun d'eux 
n'est altéré par le feu. Ce projet devra préciser les fonctions de ces outils à partir de répliques expérimentales.

D'autres sites des Némencha ont livré des documents comparablables. Je citerai d'abord le cas de Bir Sbaïkia (Sbeïkia) et la création du «Sbaïkien» (Reygasse 1921-1922; 1936-1937), également discrédité (Balout 1955, p. 454). D’identiques remarques fondées sur photos et examen d'objets triés, déposés et conservés dans les mêmes institutions, me conduisent à rester prudente, tout en m'interrogeant sur les raisons de telles potentialités industrielles régionales.

12 A une quinzaine de kilomètres à l'Ouest de Tébessa et du Djebel Mistiri, ce fut déjà M. Reygasse qui découvrit en 1917-1918 les premiers instruments bifaciaux d'Aïn el Ouksir-Tazbent (Camps 1960, 1961). Surpris par leur nombre et leur état, il en soulignait déjà le caractère fruste et rapprochait ces pièces de l'Acheuléen et du Moustérien "Sur le plateau de Tazbent, j'ai relevé, cependant, un outillage acheuléomoustérien qui differe essentiellement de toutes les industries analogues signalées... en effet, les coups de poing acheuléens sont associés à d'énormes grattoirs concaves et à des rabots de grandes dimensions. » Voilà pourquoi, lorsqu'il découvrit plus tard, dans l'Oued Mahrouguet, tant d'instruments semblables, il n'hésita pas à créer le «Faciès Mahrouguétien ».

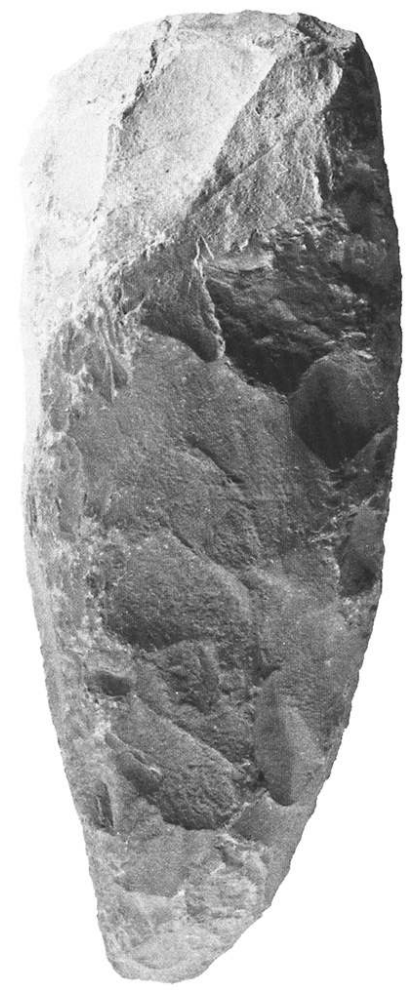

Fig. 4. cognée de bûcheron, en SILEX, PROVENANT du dAMOUS EL-AHMAR (SUd de tÉbeSSA) et de SON CONTEXTE NÉOLITHIQUE DE TRADITION CAPSIENNE. CETTE PIÈCE MESURE 213 MM DE LONgUEUR MAXIMUM ET 83 MM DE LARgEUR MAXIMUM.

Cl. F.-E. Roubet, 1965, (coll. CNRPAH, Alger).

13 Aujourd'hui, en raison de sa localisation à proximité du " quadrillage de Tazbent », qui fait l'objet d'un nouveau projet d'étude, cette collection prend un nouvel intérêt (Fig. 2, 3 , 4) (Roubet 2008, Aouras n ${ }^{\circ}$, sous-presse). 

Néolithiques et surtout durant l'époque proto-historique, dans les Némencha, passe par le réexamen de sites et de leur nombreux instruments. Pour les premières houes maghrébines du Mahrouguétien, les comparaisons les plus pertinentes peuvent se faire, par défaut, avec des instruments d'un faciès agricole africain, para-Toumbien (Vaufrey, 1969, pl. XIII, $\mathrm{n}^{\circ}$ s 1 et 3 ; pl. XIV, $\mathrm{n}^{\circ}$ s 4 et 6 ); et pl. XV, $\mathrm{n}^{\circ}$ 13), et celles du Fayoum, en Egypte.

De nouvelles perspectives de recherches se dessinent. Ce nouveau projet devrait rassembler les éléments majeurs d'une documentation abondante et variée, un peu oubliée et incomprise. Une réévaluation devrait permettre à la Néolithisation atlasique finissante de se prolonger encore, voire de s'accomplir en s'impliquant dans le domaine agricole, et, à la notion de « terroir berbère des Némencha » de se définir.

\section{BIBLIOGRAPHIE}

BALOUT L., 1955 - Préhistoire de l'Afrique du Nord. Essai de chronologie, Paris, AMG, 544 p. (Mahrouguétien : p. 5, 14, 28, 134, 152, 387, 452, 458, 463 ; Sbaïkien p. 454-458).

CAMPS G., 1960 - Aux Origines de la Berbérie. Massinissa ou les débuts de l'Histoire. Libyca, ArchéologieEpigraphie, Bull. du Service des Antiquités, t. VIII, 1 er semestre, 320 p, (Tazbent : p. 72-77).

CAMPS G., 1961 - Aux Origines de la Berbérie. Monuments et rites funéraires proto-historiques, Paris, AMG, 628 p, 1 dpl. (Dj. Mistiri et Dj. Tazbent : p. 579).

Debruge A., 1909 - « La Préhistoire dans les environs de Tébessa », Rec. des Not. et Mém. de la Soc. archéol. de Constantine, t. XLIV, p. 53-100.

REYGASSE M., LATAPIE M., 1911 - « Découvertes préhistoriques dans le Cercle de Tébessa », Rec. des Not. et Mém. de la Soc. archéol. de Constantine, t. XLV, p. 351-354.

REYGASSE M., 1917-1918 - « Observations sur les techniques paléolithiques du Nord-NordAfricain», Rec. des Not. et Mém. de la Soc. archéol. de Constantine, t. LI, p. 275-282.

REYGASSE M., 1921-1922 - «Etudes de palethnologie maghrébine-2è série », Rec. des Not. et Mém. de la Soc. archéol. de Constantine, t. LII, p. 159-204.

REYGASSE M., 1934 - « Découverte d'une technique campignienne dans le Paléolithique inférieur du Sud-Constantinois ", XI Congrès Préhist. de France, Périgueux, p. 570-573. 
REYGASSE M., 1934 - « Sur une industrie campignienne découverte dans la région de l'Oued Mahrouguet (Sud de Tébessa) », Journ. de la Soc. des Africanistes, t. IV, p. 115-116.

REYGASSE M., 1936-1937 - « Notes sur la distribution géographique et la morphologie de diverses stations préhistoriques relevées sur le territoire de la Commune mixte de Tébessa (SudConstantinois) », Bull. de la Soc. de Préhist. et d'Archéol. de Tébessa, t. 1, p. 29-117.

ROUBET C., 2003 - « Statut de berger » des communautés atlasiques néolithisées, du Maghreb oriental », L'Anthropologie, t. 107, p. 393-442.

ROUBET C., 2006 - «Pastoralisme et ruralité néolithiques dans l'Aurès, avec la grotte Capéletti, entre $7500 \mathrm{cal}$ BP et $5400 \mathrm{cal}$ BP », Aouras, n 3, p. 19-35.

VAUFREY R., 1969 - Préhistoire de l'Afrique. Tome II Au Nord et à l'Est de la Grande Forêt, Publ. Université de Tunis, vol. IV, $372 \mathrm{p}$.

INDEX

Mots-clés : Agriculture, Algérie, Capsien, Néolithique, Préhistoire 